\title{
Repositorios públicos frente a la mercantilización de la Ciencia: apostando por la ciencia abierta y la evaluación cualitativa
}

\section{Public repositories against the commodification of Science: betting on open science and qualitative evaluation}

\author{
Jorge Caldera-Serrano \\ jcalser@alcazaba.unex.es \\ Universidad de Extremadura
}

\section{Resumen}

Se lleva a cabo una reflexión sobre la necesidad de aportar y difundir la investigación financiada con fondos públicos por medio de repositorios institucionales, los cuáles servirían además como método de evaluación de la Ciencia, eliminando de esta manera la tiranía del factor de impacto como método de evaluación de la transmisión del conocimiento, e incorporando políticas públicas con leyes transparentes que modifiquen la incursión de plataformas privadas (principalmente WoS y Scopus) como elementos para la evaluación de la ciencia. Se parte de la premisa de que los autores difunden los contenidos en revistas de acceso restringido (pago por acceso) derivado de la falta de valoración institucional de los repositorios públicos como instrumento probatorio de calidad y méritos, por lo que se facilita un método alternativo en el marco de la filosofía del Open Access, para valoración y muy especialmente la difusión de las investigaciones. Para ello se analiza la validez de los repositorios y los cambios necesarios en ámbitos autonómicos, estatales como europeos.

\section{Palabras clave}

Evaluación de la ciencia; Políticas científicas; Repositorios institucionales; Open Access. 


\section{Abstract}

A reflection is carried out on the need to contribute and disseminate financial research with public funds for the medium of institutional repositories, the projects also serve as a method of evaluating science, thus eliminating the tyranny of the impact factor as Method of Evaluation of the transmission of knowledge, and incorporation of public policies with transparency that modify the incursion of private platforms (mainly WoS and Scopus) as elements for the evaluation of science. The part of the premise that the authors disseminate the contents in the restricted access journals (derived from the lack of approval of the documentation) of the public repositories as a quality and merit evidence tool, for which an alternative method is provided in the framework of the philosophy of open access, for assessment and, especially, the dissemination of research. For this purpose, the validity of the repositories and the necessary changes in the autonomous, state and European sphere are analyzed.

\section{Keywords}

Science evaluation; Scientific policies; Institutional repositories; Open Access.

Recibido: : 6/09/2018

Aceptado: 26/11/2018

DOI: https://dx.doi.org/10.5557/IIMEI9-N17-074101

Descripción propuesta: Caldera-Serrano, Jorge, 2018. Repositorios públicos frente a la mercantilización de la Ciencia: apostando por la ciencia abierta y la evaluación cualitativa. Métodos de Información, 9(17), 74-101

\section{Introducción}

"A finales del siglo XX algunos pensadores habian afirmado que con la caída de la Unión Soviética se definia un nuevo orden político en el que la forma de gobierno basada en la democracia liberal y en una economía de libre mercado capitalista permitían hablar del fin de las ideologías. De esta forma, el liberalismo democrático sería la estación de llegada final para la sociedad mundial' (Vives 2005). 
Tal y como señala el mismo autor, en la actualidad esta presunta calma no es real en el ámbito económico, siendo internet donde encontramos una de las principales discrepancias. La Economía y los mercaderes (dueños del Mercado) intentan continuamente impedir una de las grandes metas, un gran sueño factible, como es el acceso a la información de manera universal. Y la gran excusa, el principal obstáculo, es la propiedad intelectual. Autores como Muela (2004) han definido el concepto de "liberación de la información" como modelo de superación de "libertad de la información" (Vives 2005). Y aunque se ha demostrado que existen modelos generados por el propio autor para facilitar las obras, como el movimiento Open Access, en el que el concepto de "open" debe interpretarse como libre o gratuito, el Mercado y su maquinaria siguen poniendo grandes inconvenientes a la difusión libre de la información. La Información es Mercado al ser ésta poder, y por lo tanto un bien comercializable (Suber 2005).

Y siguiendo la línea del autor anterior, Torres (2011) reseña el cambio en la década de los 70 del siglo $\mathrm{XX}$, un cambio en el modo de producción en el sistema capitalista: la transformación de una economía industrial a otra informacional. Y que la Información ha generado cambios en los métodos "de producción” derivados del cambio de materia prima (la información). Tal y como señala Castell $(2000,2001)$ la actividad científica y tecnológica es igualmente una actividad destinada a la difusión de dicha materia prima; es decir, no tiene cabida la actividad científica, si no es con el fin de difundir los descubrimientos. Y en el marco de dicha producción científica, en nuestro contexto social, y teniendo presente que la difusión debe ser clave y premisa, se han acuñado términos como "ciberinfraestructura", "e-ciencia", "einvestigación" (Foster 2005, Hey 2005) que analizan otras nuevas maneras de difusión del conocimiento científico. Torres (2011) identifica que gran parte de las investigaciones en materias como las Altas Energías (Física), Ecología, Medio Ambiente, Biología Molecular, Geología, por citar algunas áreas que generan conocimiento a partir de programas públicos, con financiación enteramente pública, por lo que los resultados que de ellos derivan debieran ser propiedad de todos. ¿Todo lo publicado con fondo público es accesible para la ciudadanía que lo paga?, sin duda que sí.

Torres (2011) defiende, con gran acierto -entendemos- que "la idea central es que los datos generados por financiamientos públicos son patrimonio de la bumanidad y 
deben estar accesibles y disponibles tan amplia y directamente como se pueda", asentando dicha afirmación en autores como Arzberger (2004), Lessing (2004) y Alonso (2006). Esto contrasta con la visión errónea de muchos investigadores, que estiman que el resultado de su trabajo es personal, o del grupo de investigación, y que por lo tanto la forma de difusión y rentabilidad es elegida por ellos. Esta idea, junto con las restricciones de los editores, hace que la investigación con fondos públicos no sea fácilmente accesible (Torres 2011). Seguramente no todo deba ser accesible, seguramente habrá que analizar elementos que comprometan la difusión, pero los derechos editoriales cedidos por autores que han obtenido resultados con fondos públicos no deberían tener cabida (Murray-Rust 2008).

\section{Objetos, objetivo y metodología}

La multiplicidad de las aristas de lo planteado en este estudio hace un tanto complicado el marcar un solo objeto de análisis y valoración. Partimos de la necesidad de valorar la situación actual sobre la evaluación de la actividad científica en España, de escudriñar cómo diferentes políticas científicas han llevado al sistema estatal a una parálisis derivada del estancamiento institucional y del parón económico en la inversión en $\mathrm{I}+\mathrm{D}+\mathrm{i}$. No obstante, este trabajo no pretende centrarse en cómo se ha llegado hasta esta situación y quiénes han sido los que han decidido denigrar nuestro sistema de investigación consiguiendo que un número importante de investigadores dejen el país y otros simplemente hayan engrosado las ya abultadas listas de desempleados. Las políticas de los partidos estatales que hasta hoy han estado en el poder, han visto siempre la financiación a la Ciencia como un gasto y nunca como una inversión. Partido Popular (PP) y Partido Socialista Obrero Español (PSOE) en poco se han diferenciado en materia de inversión en investigación, siendo siempre una partida de la cual se han podido esquilmar recursos.

Se han creado además desde las instituciones políticas estatales organismos como la ANECA, a la cual le han otorgado un poder excesivo sobre todo el sistema universitario y de investigación, basando sus evaluaciones de profesorado -especialmente- en criterios alejados realmente a la calidad individual de los trabajos aportados. 
Derivado de esta situación caótica y crítica, el objeto de trabajo podría entenderse múltiple, ya que se analizan y se interrelacionan la evaluación científica, los repositorios institucionales como respuesta o, al menos, alternativa sobre y para la evaluación de la Ciencia, y todo ello aderezado por la necesaria implementación de políticas científicas para un correcto desarrollo. Estos objetos se deben relacionar para implementar un nuevo sistema, el cual difícilmente puede resurgir desde el sistema actual, y que necesita una clara revolución en las instituciones que actualmente se encargan de esta labor. Y esta revolución no puede venir de las instituciones universitarias o de investigación, aunque puedan potenciarla, sino que debe venir de apuestas políticas claras y tajantes, donde se le otorgue la importancia al contenido de la investigación y no a la fuente de difusión.

Desde este trabajo se apuesta por "nacionalizar" la ciencia, que vuelva a ser pública, controlada por lo público, y gestionada y evaluada desde lo público. No obstante, ya adelantamos algunas conclusiones, y es que ante la falta de interés mostrado de forma reiterada por llevar a cabo estos cambios en nuestro país, estimamos que será desde las instituciones de la Unión Europea desde donde debe comenzar el cambio; que impregne, por medio de la obligación legislativa, la forma de entender la Ciencia, de devolverla a la sociedad, y que deje de ser parte de la Economía y de intereses privados.

El objetivo, por tanto, parece más evidente: mostrar una alternativa centrada en los repositorios institucionales (regionales -europeos- o nacionales) como fórmula de valoración de la producción y datos de investigación científico y técnica, y de la evaluación de la ciencia en general. Y todo ello teniendo presente cambios en las políticas (autonómicas, nacionales e internacionales), en las técnicas (poniendo al repositorio y al individuo,) como centro de la valoración de investigación, dejando de lado el factor de impacto como único método de evaluación) y en los investigadores, los cuales debemos y podemos presionar para un cambio en la dirección de la socialización y universalización del conocimiento, devolviendo el patrimonio del conocimiento a la sociedad.

La metodología empleada no deja de ser un clásico en las ciencias sociales: la revisión bibliográfica junto con la valoración socioeconómica y política de la sociedad. Evidentemente tras este enfoque existe una apuesta, una visión 
europeísta y de izquierdas para entender la Ciencia, una apuesta por recuperar la lucha desde la izquierda política para favorecer a los de "abajo".

\section{Repositorios institucionales al amparo del Open Access}

La iniciativa Open Access ha modificado la forma de difusión del conocimiento científico, específicamente de la publicación y puesta a disposición de la información a la comunidad científica. La Declaración de Budapest orienta las estrategias para el desarrollo del Open Access por medio de dos vertientes: el primero, la publicación en revistas en abierto, ya sea de nuevo cuño, o la adaptación de las antiguas a este nuevo modelo; y por otro lado, la creación también de repositorios como fórmula de difusión del contenido científico. A este segundo mecanismo de implementación de la filosofía de la distribución de contenido en Open Access se le denomina "ruta verde" (Sánchez 2017). Este mismo autor identifica las características de los repositorios en acceso abierto: deben contar con la tecnología de una escritura y muchas lecturas (WORM); coste bajo de mantenimiento; el software debe contener licencia de código abierto (open source) para que se pueda adaptar a las necesidades específicas; las normas del repositorio deben ser conocidas y públicas; la actuación de los recursos debe ser rápida, y la difusión debe ser cuantificable.

Ya son varias décadas lo que se lleva debatiendo la necesidad de facilitar en abierto las investigaciones (data sharing), lo que es un pilar fundamental para la e-ciencia como instrumento de carácter acumulativo del saber (Shneiderman 2008). Torres-Salinas (2012) otorga grandes ventajas a la adopción de dicha actitud por parte de los investigadores. Son muchos y contrastados los beneficios de la difusión en abierto de los datos y resultados de la investigación (Arzberger et al. 2004, Vickers 2006). Sin lugar a dudas, el rendimiento económico es un factor primordial; parece oportuno y necesario en un contexto de crisis o simplemente de optimización de recursos que debe optimizarse el dinero invertido en generación de conocimiento científico, sobre todo de aquellas investigaciones financiadas con dinero público. La difusión de esta información ayudaría a llevar a cabo metaanálisis de la ciencia (Ramasamy et al., 2008) así como ahorro por medio de control de la investigación de los diferentes grupos (de esta manera no se solaparían las investigaciones, y las redes y colegios invisibles serían más importantes y 
activos). Para los autores supondría un aumento en la visibilidad y citación de sus trabajos (Piwowar, Day, Fridsma 2007).

Torres-Salinas (2012) señala que el primer repositorio aparece en 1971 con la creación del Protein Data Bank (PDB) bajo los auspicios de la American Crystalographic Association (Crawford, Hurd y Weller 1996, Berman, 2007). Aportamos algunas definiciones y puntos de vista: "Los repositorios institucionales, también conocidos como repositorios digitales, están constituidos por un conjunto de archivos digitales en representación de productos científicos y académicos que pueden ser accedidos por los usuarios" (Texier 2013). Este mismo autor señala que los repositorios institucionales tienen el propósito de recopilar, catalogar, gestionar, acceder, difundir y preservar, siendo necesario realizar las labores de catalogación, acceso, gestión y difusión de los contenidos, al igual que la recopilación y preservación. Los dos últimos elementos aún siguen siendo los principales problemas.

Otras definiciones son las aportadas por Texier (2013) de diferentes autores, que están presentes desde la introducción del concepto por parte de Crow (2002), quien lo definía como colecciones digitales para la captura y preservación de la producción de las comunidades universitarias, específicamente. Un año después, Lynch (2003) también señala que un repositorio debe contar con el compromiso de la organización y de los miembros de dicha institución, ya que de lo contrario su función se resquebrajaría. Van de Sompel et al. (2004) nos presenta claramente que la difusión de contenidos en repositorios es una forma de registrar la evolución académica de una institución, pudiendo así analizar tendencias y líneas de futuro. Tramullas y Garrido (2006) nos indican que los repositorios institucionales se han convertido en la principal forma de publicación y preservación, además de la difusión, de información digital, gracias a la unión del acceso abierto, software libre y estándares abiertos. Bustos y Fernández (2008) inciden en el concepto de la preservación y difusión de material en formato digital, desde un portal web accesible, por lo que plantea una forma inmejorable para la difusión de contenidos científicos, pero no como fórmula de publicación.

Esta discusión parece ser permanente, el que los repositorios puedan entenderse como método de publicación o simplemente de difusión. Los 
mecanismos para que evolucionen son muy sencillos desde el punto de vista formal, ya que identificando como revista y aportando los criterios de calidad propios de las mismas, podría entenderse como método de publicación (si es que actualmente el concepto de publicación y difusión no están fundidos en uno), el problema es la visibilidad y validez institucional que buscan muchos autores y científicos en los formatos de difusión de sus trabajos.

Tramullas y Garrido (2006), citando a Crow (2002) identifica los cuatro elementos básicos que definen a un repositorio institucional: definido y establecido institucionalmente; el contenido es académico o científico; su carácter es acumulativo y perpetuo; y es de acceso abierto.

Las fases para la implantación de un repositorio son marcados por diferentes autores, destacamos un caso real (Alonso, 2008). Una primera fase con revisión bibliográfica para analizar el marco teórico, así como se entiende fundamental el analizar la legislación española y europea y los protocolos de intercambio de información; una segunda fase en la cual se identificaría el software necesario; una tercera etapa en la que se programa y configura el sistema atendiendo a las necesidades; y la cuarta fase en la cual se valida e implementa propiamente el repositorio.

Estos mismos autores (Alonso, 2008) señalan que para que pueda implantarse de manera correcta y efectiva un repositorio institucional es necesario contar con una política clara e institucional de acceso abierto, así como una estrategia económica, tecnológica y legal. No existe una normativa clara sobre la transferencia de información en acceso abierto; trabajos que analizan la cesión voluntaria de estos trabajos por parte de los autores han llegado a la conclusión de que no es efectiva. Los autores prefieren difundir por otros canales antes que por repositorios, derivado del entendimiento de que las revistas de pago cuentan con mayor prestigio, lo que ponemos en duda ya que existen intereses económicos por encima del prestigio. Ahora bien, lo que está claro es que existe una mayor visibilidad si se incluye la información en repositorios gratuitos, e incluso mayor citación, aunque dicho aumento de la citación no tiene que tener una clara correspondencia en los índices de impactos de las empresas privadas. La realidad que señala Harnad y Brody (2004) es que las políticas e iniciativas de acceso abierto no son bien recibidas 
por los investigadores ya que se tiene la percepción de que no es la mejor fórmula para difundir la investigación para que tenga repercusión e impacto.

El Consejo de Europa publicó el 10 de enero de 2007 (European Research Council, 2007) las directrices sobre el Open Access:

"The ERC requires that all peer-reviewed publications from ERC-funded research projects be deposited on publication into an appropriate research repository where available, such as PubMed Central, ArXiv or an institutional repository, and subsequently made Open Access within 6 months of publication". "The ERC considers essential that primary data - which in the life sciences for example could comprise data such as nucleotide/protein sequences, macromolecular atomic coordinates and anonymized epidemiological data - are deposited to the relevant databases as soon as possible, preferably immediately after publication and in any case not later than 6 months after the date of publication".

Importante medida que obliga a facilitar la información a repositorios en acceso abierto, siendo el tiempo de embargo relativamente corto; no obstante no está funcionando en estos momentos como realmente debiera, ya que queda circunscrito a material procedente de proyectos financiados

\section{La Declaración de San Francisco (DORA) como camino y fin.}

Con el fin de mejorar la forma en la que se mide los resultados de la investigación, en diciembre de 2012, un grupo de directores y editores de revistas académicas se reunió durante la Reunión Anual de la Sociedad Americana de Biología Celular, en San Francisco (Estados Unidos), de ahí surge la declaración de San Francisco o DORA por su acrónimo en inglés (Declaration on Research Assessment).

En dicho documento señala que es "imperativo que la producción científica se mida con precisión y se evalúe con prudencia".

Nos recuerda que el índice de impacto, factor determinante para la evaluación en la ciencia en el contexto actual, tenía la finalidad inicial de ayudar a los bibliotecarios a decidir cuáles eran las revistas que se debían adquirir para sus bibliotecas, atendiendo al uso de las mismas (no a la calidad, la cual no se relacionaba con el impacto). 
DORA (2012) entre otras cuestiones señala que "los índices de impacto de las revistas no son ni transparentes ni están abiertamente a disposición del público", utilizando para ello como base los trabajos de Vanclay (2012) y Rossner, et. al $(2007,2008)$. Las recomendaciones de DORA desean que los resultados de la investigación que no sean presentados en forma de artículos comiencen a tener valor e importancia, siendo la 'evaluación por pares' la tendencia para evaluar la validez del trabajo científico.

Las recomendaciones tienen una validez tanto para las universidades y otras instituciones académicas y de enseñanza superior, como para revistas científicas, para las organizaciones que analizan la ciencia y para los políticos que las regulan, y muy especialmente para los autores, a los cuáles se les pide un cambio de estrategia y de orientación en la forma de comunicar su investigación.

Las recomendaciones se basan en: a) la eliminación del uso de las métricas basadas en revistas para la toma de decisiones, b) la evaluación de la investigación por sus méritos y no por el método y fuente de difusión, y c) aprovechar las oportunidades de la publicación en línea y en abierto (explorando nuevos indicadores).

Además, solicita que se sea claro y explícito en los criterios por los cuáles se evalúa el trabajo de investigación, señalando claramente que lo importante de un trabajo de investigación es el contenido y no la fuente de publicación.

Anima a los editores a utilizar la publicación en línea, y entendemos que gratuita, para una mayor difusión de la Ciencia, sin llevar a cabo restricciones en el número de referencias. Apuesta por la publicación de los trabajos bajo la licencia Creative Commons Public Domain Dedication.

Y para finalizar, exhorta a los investigadores a que cuando formen parte de comités de 'toma de decisiones' realicen sus evaluaciones a tenor del contenido, que use otras medidas para evaluar los trabajos, y que promueva prácticas centradas en la calidad del contenido.

El Grupo Parlamentario Confederal Unidos Podemos-En Comú Podem-En Marea presentó una Proposición No de Ley (PNL) relativa al actual sistema de 
evaluación y acreditación del profesorado universitario por parte de la ANECA. Dicha proposición no prosperó.

Esta PNL (2017) señala que existe una fórmula anómala de analizar la producción científica, basada en elementos externos al propio trabajo y sí relativa a la fuente, por lo que se valoran las fuentes de publicación y no tanto el contenido de la misma, lo que debería ser el elemento a evaluar. Creemos que de la PNL, que sigue el documento DORA, es muy crítico con el factor de impacto, además de no estar de acuerdo en la premisa de que la única forma de difundir la investigación sea por medio de la difusión en revistas concretas.

La decisión de que el factor de impacto es el método de evaluación es una decisión política, basada en datos de dos multinacionales extranjeras, a las cuáles se les paga mucho dinero por aportar estos datos. Dos empresas privadas evalúan la calidad con criterios no tanto científicos como empresariales. Clarivate, con su producto Web of Science, y Elsevier, con su producto Scopus (especialmente la primera) son las herramientas seleccionadas para evaluar la calidad de la producción científica, con los muchos sesgos y problemas que se han planteado desde la literatura científica.

Al ser muchas de estas revistas de pago (ya que un número importante de ellas son de la propia empresa, en el caso de Elsevier) y básicamente en inglés, hace que muchas de las investigaciones pagadas con dinero público se difundan y se cedan los derechos a las revistas integradas en estas plataformas multinacionales. Y los autores no se plantean otras opciones derivado de que la evaluación de su actividad investigadora (los tramos de investigación) realizada por la CNEAI (de ANECA) como el ascenso en las diferentes figuras contractuales de la universidad, se fundamenta en la evaluación del impacto de las revistas. El índice $\mathrm{H}$, como el Google Scholar Metrics, tampoco mejoran la evaluación, ya que al final también se basan en los mismos criterios de impacto.

No publicar en inglés está penalizado, al tener menor posibilidad de publicación en estas revistas, lo que debe ser corregido por decisiones políticas. 
Hacemos nuestro los postulados de DORA y de la Declaración de Berlín, de ahí que propongamos un nuevo sistema de evaluación, lo que se traduce además en cambios legislativos básicos y fundamentales para que puedan realizarse estos cambios para el bien, no sólo de la evaluación de la actividad científica, sino para una socialización de verdad del conocimiento científico entre los propios investigadores y entre la sociedad, la cual paga en muchos casos las investigaciones, por medio de proyectos concretos, al igual que paga el salario de los investigadores del sistema público. Un doble pago que, como gran anomalía, después acaba en empresas privadas para su difusión, mismas herramientas que después son tenidas en cuenta por nuestros dirigentes políticos para evaluar la producción de nuestros investigadores.

\section{5. ¿Cambiamos el modelo de difusión y de evaluación de la actividad científica?}

Tal y como Señala Zischman (1972) la comunicación científica se considera como la esencia misma de la ciencia. Difundir para discutir, discutir para avanzar y mejorar, poner a disposición de la comunidad científica da sentido al carácter acumulativo de la Ciencia y a la evolución del conocimiento. Y las revistas son actualmente las grandes protagonistas en la difusión de la información, incluso en áreas como las Ciencias Sociales y las Humanidades, que tradicionalmente han utilizado la monografía y la literatura gris, especialmente actas de congreso (Lariviere et al. 2006). El artículo en revista científica es el método más rápido para poner a discusión un trabajo, además de entenderse como un reconocimiento y una manera de darle credibilidad y validez al contenido del trabajo, al dar validez a la revisión por pares como método de la evaluación de valía científica. Además, la revista se ha tomado como el formato preponderante para la evaluación de la investigación (de ahí, seguramente, el cambio en la forma de difusión por parte de diferentes áreas y disciplinas).

Aunque apostemos por integrar otros tipos documentales, volvemos a recordar que la evaluación de la Ciencia, básicamente en forma de artículos científicos, se basa en el factor de impacto. Son muchos los trabajos que critican este factor como mecanismo de evaluación, por lo que destacamos únicamente el trabajo de Cañedo et al. (2005) para que sirva de referencia 
(úsese el aparato crítico para ampliar visiones) para valorar el factor de impacto.

El factor de impacto cuenta con importantes limitaciones y se llevan a cabo numerosos abusos en su nombre (como las evaluaciones de calidad de la producción científica por parte de la ANECA).

Un elemento fundamental criticado es el sesgo de las revistas analizadas para determinar el índice de impacto. La selección es realizada por empresas privadas, con criterios nada transparentes. La cobertura de las revistas cubre de forma privilegiada el ámbito anglosajón en idioma inglés, siendo mal tratadas otras zonas geográficas y otras lenguas. Esto se traduce en que los artículos más citados son los publicados en revistas norteamericanas e inglesas.

Otro factor fundamental es que los elementos que se utilizan para identificar el factor de impacto en nada tienen que ver con la calidad de los artículos.

Uno de los criterios para incluir las revistas en estos índices es que hayan sido citados, de tal manera que revistas que estén fuera de esta citación por muy buenas que sean no estarán presentes ni a corto ni medio plazo (y seguramente nunca). Campos y temáticas novedosas, y por lo tanto las revistas, no son citadas ni refrendadas como frentes de investigación (hay menos revistas, y por lo tanto menos artículos para citar y citarse).

Una revista, por muy buena que sea, no es posible que la calidad de todos los trabajos sea la misma. Por lo tanto, extrapolamos criterios para el análisis de fuentes (revistas sobre todo) para el análisis de la calidad de los trabajos de investigación.

Otros elementos que pervierten los resultados son las autocitas, las revistas que incitan a la autocitación, la tendencia de los autores a citar a los ya citados para no tener problemas con los revisores (efecto Mateo), etc.

Por todos estos problemas técnicos, éticos y filosóficos que plantea el factor de impacto, plantearnos un repositorio como mecanismo de evaluación, recopilación y difusión (además de preservación) de colecciones de datos y 
publicaciones científicas no es nuevo. Existen importantes y más o menos exitosos ejemplos de repositorios institucionales como Open Scholar (https://www.openscholar.org.uk/) e iniciativas como Dialnet, Redalyc, SciELOe incluso Latindex que han intentado corregir el sesgo geográfico e idiomático de las herramientas tradicionales y utilizadas para la evaluación. Plantearnos esta idea, ayuda a entender el valor de la ciencia cooperativa, limando la competitividad que en la actualidad se potencia. Igualmente ayuda a no vender el conocimiento científico como producto del mercado, quedando como bien general para el aprendizaje colectivo. Y en último lugar, es una forma de transferir a la sociedad el conocimiento que gracias a ellos se ha conseguido.

Por lo tanto, la propuesta planteada, tal y como marcaba la PNL descrita con anterioridad, es la puesta en marcha de un "Nuevo Sistema Público de la Evaluación de la Producción Científica en Abierto" (utilizando la denominación realizada por los grupos parlamentarios, aunque sólo lo circunscribían a nivel nacional). Sistema que no puede estar en manos de un organismo autónomo como la ANECA en el caso español, sino que debe ser recuperada por instituciones públicas como la FECYT. Este sistema, además, debe tener ramificaciones en los departamentos de investigación de las comunidades autónomas, donde se implantarán y se controlarán los repositorios bases autonómicos, repositorios que serán las herramientas fundamentales para evaluar a los investigadores y para sus potenciales mejoras salariales.

Repositorios que analicen todos y cada uno de los tipos documentales, no solo artículos, integrando igualmente formatos derivados de la literatura gris (actas de congresos, informes, tesis doctorales), informes de investigaciones en curso, y otros formatos que integren los saberes no sólo de lo que denominamos ciencias puras, sino también de las ciencias humanas y de las ciencias sociales.

La Unión Europea apuesta teóricamente por el cambio de paradigma en la difusión de los trabajos científicos y por el trabajo colaborativo, lo que se traduce en la creación de un Espacio Europeo de Investigación único, que potencie la relación y la cooperación transfronteriza. 
Su inversión en investigación es clara, ya que en 2014 puso en marcha el programa Horizonte 2020, el cual está dotado de 80.000 millones de euros (2014-2020) de forma directa, más lo que pueda recabar de instituciones privadas y de organismos públicos de carácter nacional. Horizonte 2020 ha financiado proyectos en los que los avances son productos viables $\mathrm{y}$ comerciales, al contar con relación clara con el sector privado, además de potenciar la cooperación internacional, incluso con países no miembros de la Unión Europea.

La Comisión Europea lleva apostando por el acceso abierto desde el año 2006. En el año 2007 se presentó a la Comisión una petición para garantizar el acceso gratuito a los resultados de los proyectos financiados con fondos de la Unión Europea, lo cual fue avalado y firmado por más de 28.000 instituciones y personas relacionadas con la investigación, la universidad y el desarrollo.

Desde el 2010 la Comisión financia el proyecto OpenAIRE (Infraestructura de Acceso Abierto para la Investigación Europa) que apoya a otras instituciones para implantar el acceso abierto, suministrando infraestructura. Aún en desarrollo, trabaja con las publicaciones generadas de los proyectos 7PM (Séptimo Programa Marco de Investigación y Desarrollo Tecnológico de la Comisión Europea), garantizando el acceso abierto a material financiado públicamente. El Consejo de Europa de Investigación (ERC) publicó unas directrices en materia de difusión de contenidos de la investigación.

La Comisión ha propuesto diferentes líneas para potenciar la difusión científica generada en Europa (European Commision, 2012 a) (European Commision, 2012 b), con el fin de garantizar la difusión de los contenidos de investigación financiados con fondos públicos. Está actualizando la difusión de los contenidos del programa 7PM, como se indicó con anterioridad, no obstante además quiere igualmente difundir la producción generada por el programa Horizonte 2020. Además, desde la Comisión se pretende seguir financiando proyectos nacionales y transnacionales relacionados y que potencien el acceso abierto.

A nivel estatal, siendo optimista, lo mejor que tenemos es el mucho margen de mejora. En la Ley 14/2011 de la Ciencia, la Tecnología y la Innovación se trata, en su artículo 37, de la Difusión en acceso abierto de la actividad investigadora, la cual debe estar sufragada por los Presupuestos Generales de Estado. 
El documento generado en 2014 son unas recomendaciones (FECYT, 2014), poco aceptadas para los grupos a los que iba dirigido, el cual se centra en señalar el papel tanto de las entidades financiadoras, para aquellas que las gestionan como son las universidad y centros de investigación, como para los investigadores y para las revistas científicas. Documento generado con la mejor de las intenciones, aún sigue "sin estrenar".

Y respecto a las comunidades autónomas, señalar que la Comunidad de Madrid (http://www.madrimasd.org/madrid-ciencia-tecnologia/eciencia/repositorio-emid) y el Principado de Asturias (https://ria.asturias.es/RIA/index.jsp) cuentan con normativas que potencian en cierta manera el acceso abierto de los contenidos generados por investigación. Especialmente interesante es la labor realizada en el Principado de Asturias, los cuáles han creado un repositorio institucional para fomentar la difusión en este repositorio cuando la investigación se ha realizado con financiación autonómica.

Una vez analizadas las políticas en materia de acceso abierto y de la necesidad de realizar cambios de diferente índole para que estos repositorios puedan servir para que éstos sean utilizados como elemento de valoración y difusión de la actividad científica, vamos a ir desgranando la estructura que debiera tener este Sistema y las necesarias modificaciones a nivel europeo (fundamental), explicando cómo debe integrarse los diferentes estados, en nuestro caso, España.

La Comisión Europea ha puesto las bases para el desarrollo y mejora de la difusión de contenidos en abierto, ahora falta la segunda fase, un segundo momento de cambio legislativo en el que se comiencen a tomar acciones claras que apuesten y obliguen a la difusión por la "vía verde" de todos los contenidos producidos con dinero público. Algunas cuestiones que se podrían desarrollar desde la Unión Europea:

a) Sería muy recomendable que todo lo generado con los proyectos financiados por la Unión Europea estuvieran en un repositorio 
institucional, público, gratuito y accesible. Obligatoriedad legislada por normativas europeas.

b) Igualmente podría hacer normativo para todos aquellos países que desean acogerse a la financiación de los proyectos por medio de Horizonte 2020, y otros que vengan después, que sea obligatorio el controlar y difundir toda la producción generada en proyectos públicos, nacionales y autonómicos, de tal manera que si no se cumplen dicho requerimiento no se tendrá acceso a dichos recursos.

c) Financiación a los países para crear la estructura y mecanismos de actuación para generar el sistema nacional de repositorios de investigación.

d) La Unión Europea valorará para la financiación en Horizonte 2020, y cuantas ayudas pudiera haber en un futuro, aquellos trabajos que estén insertos en los repositorios institucionales públicos (europeo y de los países miembros), dejando el patrón "factor de impacto" como pieza clave y fundamental en la valoración.

e) La Unión Europea creará y mantendrá el Sistema Europeo de Repositorios de Difusión y Evaluación de la Actividad Científica, normalizando legislativamente todo el proceso, forma de actuación de los países implicados, y requerimientos.

Los cambios necesarios para la implantación de un sistema de evaluación alternativo, debe pasar (sí o sî) por el cambio en las normas europeas, que regulen y obliguen a este cambio de actitud, apostando por la "vía verde" como difusión del conocimiento, y haciendo efectiva sus diferentes documentos y filosofía. La Unión Europea tiene muy clara su visión sobre la difusión de la Ciencia, parece conveniente que comiencen a implementarse dichas propuestas con políticas.

Este sistema europeo debe imponerse a los sistemas nacionales, especialmente cuando no existe una especial sensibilidad para corregir las graves deficiencias que en materia de difusión de la ciencia existe en muchos países de la Unión (España es un claro ejemplo). Por lo tanto, apostamos porque sea desde la Unión Europea desde donde se generen las sinergias necesarias que 
"empapen" y obliguen al resto de países por medio de políticas e incentivos económicos (financiación de proyectos e investigadores, especialmente) ${ }^{1}$.

En el caso de España realmente debemos tratar la evaluación y sus políticas desde diferentes prismas, todos orientados a mejorar, sin duda, y sujetos a cambios por medio de la implementación de políticas. Dichos cambios deberían vertebrarse atendiendo a los siguientes parámetros: cambios en la FECYT, supresión/modificación de la ANECA, encaminarnos hacia un modelo colaborativo y abierto de la evaluación científica (aliviando al sistema de la tiranía del "factor de impacto") y adaptación a las políticas marcos que debieran estructurarse en la Unión Europea.

Los aspectos anteriores están claramente interrelacionados, y los cambios generados en la FECYT deben modificar sin duda las características y atribuciones en la ANECA, una institución por definición opaca y con una falta grave de democracia participativa. La FECYT debe volver a abanderar la investigación, controlar las políticas de investigación, marcar las pautas y, muy especialmente, gestionarlas y evaluarlas. Tomar el timón de todo el proceso y asumir competencias de la ANECA. Apostar por un sistema basado en repositorios dejando sin vigencia los métodos cuantitativos como único elemento de valoración y volviendo a la tradición del análisis de contenido (métodos cualitativos) para evaluar la ciencia. El programa PEP, que evalúa al profesorado para el acceso a las diferentes figuras académicas en la universidad; el programa ACADEMIA, centrado en la evaluación del acceso a los cuerpos de funcionarios; y la CNEAI, que evalúa los tramos de investigación, todos en manos de la ANECA, se basan en una evaluación "al peso", donde el factor de impacto es prácticamente el único factor que se tiene en cuenta, en contra de recomendaciones y tendencias en investigación que obliga y vampiriza las carreras investigadoras de profesores $\mathrm{e}$ investigadores. No entraremos hoy en cómo analiza y valora esta agencia los títulos, las universidades para facilitar los sellos de calidad o simplemente la validación para poder impartir determinadas carreras, centrémonos únicamente en la evaluación de las figuras contractuales, pero una de las

\footnotetext{
${ }^{1}$ En estos momentos, estamos desarrollando en otro trabajo el desarrollo de algunas de estas líneas que entendemos básicas para el desarrollo y evaluación de la Ciencia, centrándonos en su implementación en la Unión Europea.
} 
primeras acciones a tomar debiera ser su eliminación, o en su defecto, llevar a cabo una profunda remodelación que le otorgue transparencia, una dependencia orgánica lógica, y donde toda la comunidad pueda discutir los criterios por los cuáles va a ser evaluado. Y les aseguro, que el factor de impacto no es ni el único modelo ni el más aceptado. Nuestro modelo actual, genera una espiral que nos lleva al Efecto Mateo, de tal manera que aquellos autores más citados tienen más capacidad de seguir siéndolo, donde los que tienen proyectos de investigación, serán los que sigan consiguiendo financiación, dejando de lado a los investigadores nóveles y a todo su potencial. Por lo tanto, y partiendo de que entendemos que es necesaria una "enmienda a la totalidad" de la ANECA y de la estructura actual de la evaluación de la Ciencia en España, creemos que desde nuestro país debemos tomar como base la filosofía de la Unión Europea que va encaminada hacia la publicación en abierto de los procesos de investigación en repositorios institucionales, y por lo tanto (y eso también es una apuesta, aunque no tan explicitada por la UE) que estos repositorios cuenten para llevar a cabo la evaluación.

Las Comunidades Autónomas deberían ser apéndices en la estructura general de un sistema nacional de investigación, marcado como acabamos de incidir, en un sistema europeo que marca las normas y líneas estratégicas. Y por lo tanto, debe fomentar y potenciar los repositorios y la evaluación local con los mismos parámetros y directrices que el sistema europeo y español.

La estructura planteada es extremadamente simple, cuestión diferente es su implementación. Describamos los "trazos gruesos" de esta propuesta, que debemos ir perfilando en sucesivos trabajos, derivado de que algunas de las cuestiones planteadas deben ser analizadas con profundidad y detalle.

La estructura de este Sistema de Evaluación de la Ciencia cuenta con cuatro niveles distintos: nivel europeo, nivel estatal, nivel departamento/comunidad y en último lugar, nivel en las instituciones de investigación (universidad, institutos, etc.), por lo que traducido a la realidad española sería:

Nivel 1. Unión Europea

Nivel 2. Países de la Unión (España)

Nivel 3. Demarcaciones (Comunidades autónomas) 
Nivel 4. Universidad e institutos de investigación

La Unión Europea contaría con el mayor nivel legislativo, ya que tal y como se ha señalado, los países miembros debieran asumir los postulados a costa de mayor y mejor financiación de la Ciencia, así como para tener la capacidad de acogerse a ayudas europeas, base para la estructura actual de la financiación de la Ciencia.

La Unión Europea marcará la legislación y estructura, atendiendo a los parámetros señalados a lo largo de este trabajo (repositorio público, abierto, en el cual estén presentes todos los trabajos financiados con dinero público, etc.), y el resto de países y unidades dentro de estos países deberán aceptarlas como propias.

El repositorio generado a este nivel estaría conformado por trabajos generados por proyectos de la Unión Europea, tanto publicaciones como informes, los cuales deberán ser avalados por una evaluación por pares implementadas por la Unión. La simple publicación en el repositorio se entenderá como factor de calidad y no su publicación en fuentes privadas de pago o incluso en otras fuentes abiertas. La publicación previa será tenida en cuenta como factor importante, pero esta publicación no podrá traducirse en un embargo en su difusión. En definitiva, material generado por fondos públicos sería evaluado para su inclusión en estos repositorios, los cuales tendrían prioridad frente a la publicación en otras fuentes.

Además, se podrá solicitar la inclusión de trabajos que deberán ser avalados por la evaluación por pares para su ingreso, siempre y cuando ya estén presentes en repositorios de niveles inferiores del sistema (para solicitar el ingreso al repositorio de nivel 1 será obligatorio estar en repositorio de nivel 2, y así sucesivamente).

Es importante determinar que, dependiendo del nivel, la obligatoriedad de entrega y la prioridad de los repositorios sería idéntica en cada uno de los niveles. 
A nivel estatal se crearán estos repositorios institucionales, que recogerán (previa evaluación) los trabajos financiados a nivel estatal, y analizarán aquellos recogidos en los repositorios autonómicos que soliciten su inclusión.

El repositorio de nivel inferior (universidades, institutos de investigación, básicamente) tendrán todos los trabajos realizados por los miembros de estas instituciones (si cumple con los requisitos básicos requeridos por la evaluación por pares), lo cual será obligado para universidades e instituciones de investigación de carácter público. Entendemos que a este nivel los profesores de universidad y de instituto de investigación, al generar su investigación como parte de su labor en dichas instituciones, deberán facilitar igualmente estos trabajos al repositorio de su nivel.

Con esta estructura se garantiza que los trabajos con cierto nivel de calidad, avalado por la evaluación por pares, estén presentes en repositorios públicos, ya sea porque se han generado por proyectos de investigación financiados con dinero público o generado por la labor investigadora del personal de instituciones públicas.

$\mathrm{Y}$ aquí es donde estructuras como la ANECA deben adaptarse (o desaparecer), ya que la calidad la marcará el nivel del repositorio en el cual se difundan los trabajos de investigación, y no tendrá relación con el factor de impacto. Las ayudas públicas, las bonificaciones salariales derivadas de los méritos de investigación, la financiación de proyectos, etc., deberán tener en cuenta en qué nivel se encuentran los trabajos de los investigadores a financiar, y no otros criterios tenidos en cuenta hasta ahora.

Se pone el foco en la evaluación por pares para determinar la calidad.

Este sistema deberá dotarse económicamente por los diferentes niveles para que sea realmente ágil, generando transferencias rápidas entre los diferentes niveles del repositorio, si procede.

Otro elemento que debe analizarse para ver cómo dotar a la evaluación de un carácter social y democrático es si, como elemento a tener en cuenta por los evaluadores, se valora la evaluación por parte de la comunidad científica de los diferentes niveles. La evaluación y valoración colaborativa aún se está 
desarrollando, aunque es asumible como premisa para la mejora de la valoración de un trabajo dentro del sistema de ciencia.

La visibilidad y calidad debe ser garantizada por todo el sistema, avalando los niveles inferiores los superiores, y generando mecanismos para difundir la información que se incorpore a estos repositorios.

Sin lugar a dudas, el sistema planteado "grosso modo" plantea problema en la escalabilidad, en la financiación, en crear estructuras que sean rápidas para agilizar los procesos de evaluación, etc., entre otros factores internos; desde el punto de vista de la industria editorial, entendemos que las grandes editoriales científicas, las distribuidoras, en definitiva, los que hacen dinero con la difusión de la Ciencia que generalmente no generan, van a sentirse atacados, ya que el pago por contenidos de calidad debería caer de forma clara. Desde nuestra visión la Ciencia debe ser gratuita y abierta. En la estructura planteada, las revistas abiertas tienen su sentido como fórmula para mejorar y ayudar a la visualización, es decir, una vez que los contenidos estén presentes en un repositorio, y por lo tanto accesibles, podrán buscar "su sitio" en revistas ya sean privadas, abiertas, de pago o gratuitas.

\section{Conclusiones}

Se hace vital repensar y construir un nuevo modelo de resistencia, otra forma de generar conocimiento y de difundirlo, otra manera de que los investigadores se interrelacionen entre ellos y con sus trabajos. La Ciencia no puede ni debe entrar en los circuitos de rentabilidad económica, sino del bien social y de la difusión universal del conocimiento.

De ahí que el modelo de ciencia abierta sea el fin y el camino, haciendo nuestra y apostando por la Declaración de San Francisco y la Declaración de Berlín, para que se priorice la puesta en marcha de repositorios y revistas digitales accesibles libremente y gratuitas, y que sean desde estas herramientas desde las cuales se lleve a cabo la implementación de políticas nacionales y europeas para la evaluación de la actividad científica y para la puesta a disposición de dicho conocimiento a toda la sociedad. El conocimiento es Patrimonio de la Humanidad, y no una mercancía en venta. 
Evidentemente, para llevar a cabo esta labor se deben organizar y planificar repositorios institucionales públicos de calidad, que garanticen la preservación actual y futura, y los cuáles tengan mayor visibilidad y autoridad. Para ello es imprescindible un cambio en el modelo legislativo que le otorgue valor para posteriores reconocimientos profesionales. Y estimamos que es desde la Unión Europea desde donde se debe comenzar a marcar las pautas para este cambio de modelo, un cambio hacia un modelo democrático y accesible de la ciencia y el conocimiento, donde el conocimiento vuelva al pueblo, que es quien sustenta con sus impuestos la mayor parte de la subvención a la ciencia por medio de proyectos de investigación, y quien paga igualmente los salarios a la principal masa de investigadores (profesores universitarios e investigadores de instituciones públicas).

Tal y como hemos reseñado, apostamos por la creación de repositorios interconectados y coordinados a diferentes niveles, donde el factor de impacto deje de tener la supremacía y volvamos a la evaluación por pares y evaluación colaborativa como método de evaluación de la calidad de la investigación. Analicemos y evaluemos el contenido, y no la fuente donde ha sido publicada.

El acceso abierto para difusión de trabajos es mandato claramente reflejado por la Unión Europea, de forma específica en la iniciativa Horizonte 2020, así como en otras directivas, pero no acaba de haber una apuesta clara (con presupuesto asignado, con políticas que lo legislan y con normas que lo regulen) para llevar este mandato a término. No tenemos en España unos gobernantes especialmente sensibles a cambios en esta dirección, sino todo lo contrario. La ANECA cada vez más orientada al control del sistema universitario y de investigación, se centra en factores exógenos a la propia publicación, lo cual más que una apuesta por la calidad es una apuesta por un modelo de ciencia: externalizado, mercantilizado e infradotado. De ahí que nuestras esperanzas se pongan en los niveles superiores e inferiores: la Unión Europea y las comunidades autónomas.

Este trabajo es un alegato al cambio, mostrando una línea de debate y trabajo, una estrategia para implementar nuevas políticas orientadas a la difusión, a la mejora de la ciencia y al reconocimiento real de sus trabajadores. Otro modelo es posible si se llevan a cabo los cambios estructurales necesarios, y todo ello 
pensando siempre, no en las muchas aristas que se plantean en ciencia y en su evaluación, sino en la sociedad a la que dice servir y a la que realmente sirve. Vayamos hacia una Ciencia social, compartida, de intercambio, una Ciencia para el beneficio y el bien común.

\section{Reconocimientos}

Este trabajo ha sido financiado por el Gobierno de Extremadura (Consejería de Educación, Ciencia y Tecnología) y el Fondo Social Europeo dentro del plan de apoyo a las actuaciones de los Grupos de Investigación inscritos en el catálogo de la Junta de Extremadura. GR10019.

\section{Referencias bibliográficas}

ALONSO PERI, A., A. GALÁN PALACIO, P. PENÍN GONZÁLEZ, R. LAVANDERA FERNÁNDEZ Y J. ROLDÁN RODRÍGUEZ, 2008. La plataforma digital Repositorio Institucional de Asturias (RIA). BiD: textos universitarios de biblioteoconomía y documentació, 20(juny). [Consulta: 26 marzo 2018]. Disponible en: http://bid.ub.edu/20alons2.htm

ALONSO, B. y F. VALLADARES, 2006. Bases de datos y metadatos en ecología: compartir para investigar en cambio global. Ecosistemas, 15(2), 83-88. [Consulta: 26 marzo 2018]. Disponible en: https://revistaecosistemas.net/index.php/ecosistemas/article/viewFile/510/4 86

ARZBERGER, P., P. SCHOROEDES, A. BEAUlIEU, G. BOWKER, K. CASEY, L LAAKSONEN, D. MOORMAN, P. UHLIR y P. WOUTERS, 2004. An international framework to promote Access to data. Science, 303(5665), 1777-1778

BERMAN, H. M 2007. The protein data bank: a historical perspective. Acta crystallographica. Section A, 64(1), 88-95

BUSTOS GONZÁLEZ, A. y A. FERNÁNDEZ PORCEL, 2008. Directrices para la creación de repositorios institucionales en universidades y organizaciones de enseñanza 
superior. [Consulta: 26 marzo 2018]. Disponible en: http://eprints.rclis.org/13512/1/Directrices RI Espa ol.pdf

CAÑEDO ANDALIA, R., M. NODARSE RODRÍGUEZ, J.C. GUERRERO PUPO y R.E. RAMOS OCHOA, 2005. Algunas precisiones necesarias en torno al uso del factor de impacto como herramienta de evaluación científica. Acimed, 13(5), 17. [Consulta: 26 marzo 2018]. Disponible en: http://scielo.sld.cu/pdf/aci/v13n5/aci01505.pdf

CASTELLS, M., 2000. The Rise of the Network Society. Cambridge: Blackwell Publishers, Inc.

CASTELLS, M., 2001. The internet Galaxy. Oxford: Oxford University Press.

CRAWFORD, S.Y., J.M. HURD y A.C. WELLER, 1996. From print to electronic: the transformation of scientic communication. Medford: ASIS.

CROW, R., 2002. The case for institutional repositories: A SPARC Position paper. Washington DC:The Scholarly Publishing \& Academic Resources Coalition, 137. [Consulta: 14 enero 2019] Disponible: https://ils.unc.edu/courses/2014 fall/inls690 109/Readings/Crow2002CaseforInstitutionalRepositoriesSPARCPaper.pdf

DORA, 2012. San Francisco. Declaration on research assessment. Declaración de San Francisco de evaluación de la Ciencia: poniendo ciencia en la evaluación de la investigación. [Consulta: 26 marzo 2018]. Disponible en: http://blogs.ujaen.es/cienciabuja/wp-content/uploads/2013/10/dora.pdf

EUROPEAN RESEARCH COUNCIL, 2007. ERC Scientific Council guidelines for Open Access. . [Consulta: 26 marzo 2018] Disponible en: https://recolecta.fecyt.es/sites/default/files/contenido/documentos/erc scc guidelines open access.pdf

EUROPEAN COMMISSION, 2012. Recomendaciones de la Comisión de 17 de julio de 2012 relativa al acceso a la información cientifica y a su preservación (2012/417/UE). Publicado 21.7.2012. Diario Oficial de la Unión Europea L 194/34. Disponible en: https://eur-lex.europa.eu/legalcontent/ES/TXT/PDF/?uri=OJ:L:2012:194:FULL\&from=EN 
EUROPEAN COMMISSION, 2012a. Communication from the Commission to the European Parliament, The Council, The European Economic and Social Committee and the Commitee of the Regions: towards betters access to scientific Information: boosting the benefits of public investments in research. Brussels, 17.7.2012. COM (2012) 401 final. Disponible en: https://ec.europa.eu/research/sciencesociety/document library/pdf 06/era-communication-towards-better-accessto-scientific-information en.pdf

FECYT, 2014. Recomendaciones para la implementación del articulo 37 Difusión en Acceso Abierto de la Ley de la Ciencia, la Tecnología y la Innovación. . [Consulta: de marzo 2018]. Disponible en: https://www.recolecta.fecyt.es/sites/default/files/contenido/documentos/I mplantacion Art37 AccesoAbierto.pdf

FOSTER, I., 2005. Service-oriented Science. Science, 308, 814-817.

HARNAD, S. y T. BRODY, 2005. Comparing the impact of open access (OA) vs. non-OA articles in the Same Journal. D-Lib Magazine, 10(6). [Consulta: 26 marzo 2018]. Disponible en: http://www.dlib.org/dlib/june04/harnad/06harnad.html

HEY, T y A.E. TREFETHEN, 2005. Cyberinfraestructure for e-science. Science, 308, 817-821.

LARIVIERE, V., E. ARCHAMBAULT, Y. GINGRAS y E. VIGNOLA-GAGNÉ, 2006. The place of serials in referencing practices: comparing natural sciences with social sciences and humanities. Journal of the American Society for Information Science and Technology, 57(8), 997-1004. [Consulta: 26 marzo 2018] Disponible en: $\quad$ http://www.science-metrix.com/sites/default/files/sciencemetrix/publications/the place of serials in referencing practices post prin $\underline{\text { t } 0 . p d f}$

LESSING, L., 2004. Free culture. New York: The Penguin Press, New York edition.

LYNCH, C.A., 2003. Institutional repositories: essential infraestructura for scholarship in the digital age. Association of Research Libraries, 226, 1-7. [Consulta: 14 enero 2019] Disponible en: http://old.arl.org/resources/pubs/br/br226/br226ir print.shtml 
MUELA MEZA, Z.M., 2004. Liberación de la información como condición de la liberación del acceso a la información”. En: 1er Foro social de información, documentación y bibliotecas. [Consulta: 26 marzo 2018]. Disponible en: htpp://eprints.rclis.org/archive/00003623/01/26.pdf

MURRAY-RUST, P., 2008. Open data in science. Nature Precedings : hdl:10101/npre.2008.1526.1 : Posted 18 Jan 2008 : 23. [Consulta: 26 marzo 2018]. Disponible en: http://confoa08.sdum.uminho.pt/apresentacoes/peter murray rust npre200 81526-1.pdf

PNL n'. 162/000450, 2017. Proposición No de Ley presentada por el Grupo el Grupo Parlamentario Confederal de Unidos Podemos-En Comú Podem-En Marea, relativa al actual sistema de evaluación y acreditación del profesorado universitario por la ANECA (Agencia Nacional de Evaluación de la Calidad y Acreditación). Congreso de los Diputados. Boletín Oficial de las Cortes Generales. XII Legislatura. 8 de septiembre de 2017. [Consulta: 26 marzo 2018]. Disponible en: http:/ /www.congreso.es/public oficiales/L12/CONG/BOCG/D/BOCG12-D-204.PDF\#page $=27$.

PIWOWAR, H.A., R.S. DAY y D.B. FRIDSMA, 2007. Sharing detailed research data in associated with increased citation rate. Plos one, 2 (3),e308 . [Consulta: 26 2018]. Disponible en: https://doi.org/10.1371/journal.pone.0000308

RAMASAMY, A., A. MONDRY, C.C. HOLMES y D.G. ALTMAN, 2008. Key issues in conducting a metaanalysis of gene expression microarray datasets. Plos medicine, 5 (9), 184.

ROSSNER, M., H. VAN EPPS y E. HILL, 2007. Show me the data. Cell Biol, 179, 1091-1092

ROSSNER, M., H. VAN EPPS y E. HILL, 2008. Irreproducible results: a response to Thomson Scientific. J. Cell Biol, 180, 254-255.

SÁNCHEZ GARCÍA DE LAS BAYONAS, S. y R. MELERO MELERO, 2017. La denominación y el contenido de los repositorios institucionales en acceso abierto: base teórica para la "Ruta Verde". [Consulta: 26 marzo 2018]. Disponible en: http://digital.csic.es/bitstream/10261/1487/1/OA2rm.pdf 
SHNEIDERMAN, B., 2008. Science 2.0. Science, 319(5868), 1349-1350. . [Consulta: 26 de marzo de 2018]. Disponible en: http://dx.doi.org/10.1126/science.1153539

SUBER, P., 2005. Open Access, impact and demand. BMJ, 330(7500), 1097-1098.

TEXIER, J., 2013. Los repositorios institucionales y las bibliotecas digitales: una somera revisión bibliográfica y su relación en la educación superior. 11th Latin Amerian and Carribbean Conference for Engineering and Technology. [Consulta: 26 marzo 2018]. Disponible en: http://eprints.rclis.org/19925/1/LACCEI\%202013\%20-\%20Texier.pdf

TORRES L.A., L.A. NUÑEZ, R. TORRÉNS y E. BARRIOS, 2011. Implementación de un repositorio de datos científicos usando Dspace. Renata, 1(2), 101-117

TORRES-SALINAS, D., N. ROBINSON-GARÍA y A. CABEZAS-CLAVIJO, 2012. Compartir los datos de investigación: introducción al data sharing. El Profesional de la Información, 21(2), 173-184.

TRAMULLAS SANZ, J. y P. GARRIDO PICAZO, 2006. Software libre para repositorios institucionales: propuestas para un modelo de evaluación de prestaciones. El Profesional de la Información, 15(3), 171-181.

VAN DE SOMPEL, H., S. PAYET'TE, J. ERICKSON, C. LAGOZE y S. WARNER, 2004. Rethinking scholarly communication. D-Lib Magazine, 10(9) [Consulta: 26 marzo 2018] Disponible en: http://www.dlib.org/dlib/september04/vandesompel/09vandesompel.html

VANCLAY, J.K., 2012. Impact Factor: Outdated artefacto $r$ stepping-stone to journal certification?. Scientometrics, 92(2), 211-238

VIVES I GRÀCIA, J., 2005. Aspectos de propiedad intelectual en la creación y gestión de repositorios institucionales. El Profesional de la Información, 15(4), 267278

ZIMAN, J.M., 1972. El conocimiento público. México: Fondo de Cultura Económica. 